\title{
织物电子器件及系统的发展现状、科学问题、 核心技术和应用展望
}

陶肖明 ${ }^{1,2^{*}}$, 刘苏 ${ }^{1,2}$, 杨宝 ${ }^{1,2}$, 张莉莎 ${ }^{1,2}$, 马洁明 ${ }^{1,2}$, 舒琳 $^{3}$, 王飞 ${ }^{1,2}$

1. 香港理工大学智能可穿戴研究中心, 香港;

2. 香港理工大学纺织与服装学系, 香港;

3. 华南理工大学电子与信息学院, 广州 510640

*联系人, E-mail: xiao-ming.tao@polyu.edu.hk

2020-10-30 收稿, 2020-12-15 修回, 2021-01-07 接受, 2021-01-14 网络版发表

国家重点研发计划(2018YFC2000900)、香港研究资助局项目(15211016E，15200917E，15201419E)、香港创新技术基金(ITS/306/17)和香港理 工大学冠名教授基金(847A)资助

摘要 织物电子学研究对象是具有产生、传输、调制或测量等电子功能的纤维或纤维集合体. 织物电子系统通常 由织物电子器件和微电子器件混合互补集成而成。它们作为可穿戴系统的一个重要分支, 可为人类提供多种功能, 如感知、驱动、自适应、交流、自我修复、记忆、学习等, 而不影响系统本身的服用性. 本文从织物电子器件和 系统的定义出发, 阐述了不同类别织物电子器件的发展现状, 提出织物电子器件和系统的一些关键科学问题, 如功 能材料结构与性能的关系、织物电子器件和系统集成的多物理过程、评价系统及安全问题等. 并结合在研发织物 电子可穿戴系统及实施工程化产业化过程中的切身体会, 介绍织物电子器件和系统方面的核心技术及其应用前景. 重点对织物电子器件或系统在医疗健康、运动监测、智能防护及增强现实/虚拟现实4个方向的应用及其应用前 景进行分析.

关键词织物电子器件, 织物电子系统, 柔性可穿戴, 功能材料, 科学问题, 核心技术

织物电子学涉及具有产生、传输、调制或测量等 电子功能的纤维或纤维集合体. 织物电子系统是具有 电子功能的纤维、纱线、织物以及微电子系统共同构 成的集成系统，它们具有产生、传输和检测电子的功 能. 电子织物与织物电子器件及系统两种提法都是指 具有电子功能的“智能”纺织产品，不同之处是研究侧 重点和着眼点不同. 织物电子器件首先是电子器件, 实 现所需多种电子功能, 而织物只是其结构形态. 电子织 物强调的重点是织物, 很多场合中特指导电织物, 使电 连接柔性化或者织物化, 其他电子功能主要由集成外 部电子器件所提供.
织物电子经历了四代的发展. 第一代: 织物只是承 载结构, 将电子设备中刚性模块拆开, 分别以不破坏纺 织服装产品的可拆卸方式实现电子连接. 第二代: 织物 可提供电学和机械连接, 将刚性电子元件嵌人纺织品 中, 以一定方式附着在织物上. 第三代: 柔性织物器件 发展, 通过智能纤维及其集合体赋予电子功能, 将部分 传统刚性电子元件柔性化. 柔性织物器件与刚性微电 子元器件通过异质系统集成技术相结合形成杂化系统. 第四代: 全织物电子器件组成的电子系统, 该电子系统 的所有部件均由纤维材料经新型智能纺织工艺制成. 当前的研究以第三代杂化、第四代全织物系统为主.

引用格式: 陶肖明, 刘苏, 杨宝, 等. 织物电子器件及系统的发展现状、科学问题、核心技术和应用展望. 科学通报, 2021, 66: 3071-3087 Tao X M, Liu S, Yang B, et al. Recent advances, scientific issues, key technologies and perspective of textile electronics (in Chinese). Chin Sci Bull, 2021, 66 3071-3087, doi: 10.1360/TB-2020-1402 
目前得以成功应用的是第三代杂化系统，包括织物电 极、织物应变传感、织物连接件、织物天线等柔性较 大面积的器件等. 而在纤维基运算器件和储存器件等 方面进展甚微.

织物电子技术结合了纺织品的舒适性、轻盈、手 感和外观，以及电子产品的功能性、连接性和智能性, 无疑极具市场潜力. 过去 20 年里, 美国、德国、法国、 日本、韩国的政府和企业在该领域投人了大量科研资 源, 中国也进行了不少的研发, 取得初步成效. 据英国 剑桥大学预测，2022年采用智能纺织品的可穿戴产品 市场将达到 700 亿美元. 但是围绕可靠性、交叉兼容性 和标准、设备适用性、材料安全性和间接成本等方面 的挑战, 一直阻碍着许多织物电子产品的商业化.

本文从织物电子的基本概念人手，讨论了织物电 子器件的分类和发展现状, 并结合本团队的经验教训 梳理总结了目前织物电子领域中的重要科学问题、核 心技术、对织物电子未来发展趋势的展望等, 抛砖引 玉，旨在为织物电子技术进一步研发和应用的讨论提 供初步的看法.

\section{1 织物电子器件分类发展现状}

\section{1 柔性传感器}

除了具有传感特性外, 织物传感器还具有织物柔 软、耐用、生物相容和轻便的特性. 根据外在的刺激 种类, 可分为应变传感器、界面力传感器、温度传感 器、生物电极、气体传感器、液体传感器等. 其中, 应 变传感器可以将机械运动转换为电信号, 可广泛应用 于医疗、运动和机器人等领域的监测 ${ }^{[1,2]}$. 应变传感器 应具有优异的拉伸回复性能、耐疲劳性好、高灵敏 度、快速响应、快速恢复等优点. 但灵敏度和拉伸性 能之间的平衡、电阻型松弛现象、电容型信号干扰 ${ }^{[3]}$ 等问题尚未解决. 压力传感器将压力转变成电信号变 化. 目前, 大部分压力传感器的传感范围都大于 $10 \mathrm{kPa}$, 不能满足织物与人体接触的压力测量. 一般情况下织 物施加于人体的压力处于低压力水平, 如 2 级医用压力 袜的最高压力范围在3.1 4.3 $\mathrm{kPa}$ 之间 ${ }^{[4]}$, 而其他位置以 及普通服装对人体的压力则更小. 温度是人体最重要 的生理指标之一. 高灵敏度、高精度 $\left(0.1^{\circ} \mathrm{C}\right)$ 、反应时 间短、重复性好和检测范围 $\left(25 \sim 40^{\circ} \mathrm{C}\right)$ 是发展可穿戴柔 性温度传感器的重要指标. 光纤传感单元/器件虽然可 以达到上述要求, 但光电转换器件体积较大而限制了
其在可穿戴领域的应用. 将金属纤维/纱线直接嵌人织 物中, 或者在织物表面进行涂层也可以开发柔性织物 基温度传感器 ${ }^{[5,6]}$. 但这类织物基温度传感器的精度远 低于商用温度传感器. 另外, 织物基温度传感器的耐汗 液等性能也需要提高. 气体传感器可将目标气体的浓 度转换为光信号或电信号 ${ }^{[7]}$. 该类传感器应具备可选 择性、灵敏度高、响应快速、恢复迅速等特点. 与电 学传感器相比, 光学传感器具有更好的选择性和更高 的可靠性, 但是光电器件微小集成化程度较差. 另外, 气体传感器缺少校准标准/系统, 且可检测的气体种类 有限. 多功能集成传感器是未来发展的趋势, 可有效缩 小器件的尺寸并且提高纺织品的舒适度. 但这类多传 感还面临着多种信号的解耦和相互干扰等问题. 传感 器智能化涉及系统, 稍后讨论.

\section{2 柔性执行器}

执行器将外部能量转换为某种动作如机械形变, 能够在外部激励下提供机械反应(如动作或变形等). 由 于传统执行器(例如电动机、气动执行器、液压执行 器等)的体积较笨重, 噪音和硬度太大, 因此不适用于织 物电子系统的集成. 近年来, 柔性执行器得以迅速发

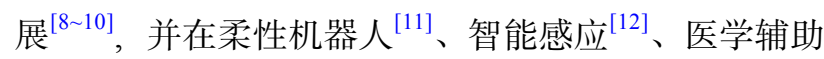
治疗 ${ }^{[13]}$ 、能量收集和转换 ${ }^{[14,15]}$ 等领域得到广泛应用. 与刚性传统执行器相比, 挠性/柔性形状促动器具有高 柔韧性、质轻和高安全性的优点, 能够通过机织缝㧅 或直接涂覆功能材料在织物上等技术集成织物执行器. 根据外部激励, 柔性执行器可分为热执行器、电化学 执行器、湿度执行器、光学执行器、形状记忆材料驱 动等.

热执行器的激励来源是热量, 当温度变化时, 执行 器的体积或形状会发生变化. 所需的热量可以通过直 接加热 ${ }^{[16]}$ 、电加热 ${ }^{[17,18]}$ 或光加热 ${ }^{[19]}$ 获得. 基于各向异 性的热膨胀行为, 半结晶聚合物 ${ }^{[8]}$ 表现出较大的热膨 胀/热收缩行为, 且这些行为是可逆的. 相变材料(如石 蜡等)受热或冷却时其体积发生明显的变化. 这些都是 理想的制备柔性执行器的材料. Copic和Hart ${ }^{[20]}$ 通过将 石蜡填充到垂直排列的碳纳米管中，研发了一个高性 能的石蜡驱动器. 该执行器能达到的最大驱动应变是 $0.02 \sim 0.2$, 这个值和肌肉的应变范围一致; 执行器的最 大应力被碳纳米管限制在约 $10 \mathrm{kPa}$. 本课题组 ${ }^{[21]}$ 最近研 究了一种基于电热效应的复合纱线执行器. 该执行器 具有优异的热驱动性能: 在 $-50 \sim 160^{\circ} \mathrm{C}$ 的温度范围内和 
$1.2 \mathrm{MPa}$ 张力下, 可实现 $20.7 \%$ 形变.

光化学执行器主要利用光敏聚合物(如偶氮苯及其 衍生物 ${ }^{[22 ~ 25]}$ )或有机分子晶体等在光刺激下分子/晶体 结构发生变化实现驱动. 这类执行器具有非接触式执 行和远程控制等独特的优势 ${ }^{[26,27]}$. 与上述基于偶氮苯 的液晶弹性体的驱动相比, 有机分子晶体的变形具有 更好的重复性、抗瘦劳性和更快的反应速度. 但是, 由 于晶体尺寸、缺陷和形状的关系, 分子晶体执行器易 碎且在性能上受到很大限制. Lan和Chen ${ }^{[28]}$ 研究了一 种基于纳米级有机分子晶体的光化学执行器. 在这种 执行器中, 管状2-羟荎酰亚胺纳米晶体有选择性地不 均匀定向分布在致密的聚偏氟乙烯-六氟丙烯共聚物 中. 在紫外线的作用下, 水平放置的纳米管弯曲成弓形, 这些弯曲的纳米管比未弯曲的纳米管占据更多的垂直 空间和更少的水平空间. 因此, 这种变形将驱动周围的 聚合物单元在水平方向上的收缩以及垂直方向上的 膨胀.

电化学执行器也称为离子执行器, 是基于离子运 动而实现的驱动. 电化学执行器具有驱动电压较低, 弹 性高且在空气中有较大的变形性等优点. 因此, 它在可 穿戴设备中具有巨大的潜力. 电化学执行器有两种工 作原理: 一种基于导电聚合物的拟电容效应, 另一种是 基于离子凝胶或离子聚合物/金属复合材料的双层效 应. 对于前一种机理, 导电聚合物(如聚吡咯及其衍生 物)在电化学反应中可失去/获得电子, 从而使聚合物链 带电. 为了保持结构的电中性, 电解质中的阴离子/阳离 子将迁移并嵌人聚合物中，从而引起聚合物的体积变 化 ${ }^{[8]}$. 对于后一种机理, 正离子和负离子在施加电压后 迁移到两个电极中, 并被吸附在电极材料的孔中以形 成双电层, 从而使电极材料膨胀. 由于正离子和负离子 的体积差异, 两个电极的膨胀程度不同, 导致执行器弯 曲 ${ }^{[29,30]}$. 用于电化学执行器的材料主要包括离子凝 胶、导电聚合物和离子聚合物/金属复合材料. 这些材 料具有质轻、柔韧性好、驱动电压低、在空气中稳定 和较大的变形能力等优点. 此外, 金属䈃、导电聚合物 和碳纳米材料常用作电极材料. Chen研究组 ${ }^{[31]}$ 设计了 平行于电场方向的石墨烯电极，该结构缩短了离子迁 移的路径，可实现高达 $98 \%$ 的可变形性，比基于碳纳米 管的执行器高 1 个数量级.

湿度执行器指基于湿度变化，材料中的亲水基团 与水分子之间的氢键发生变化，从而导致材料发生膨 胀或收缩原理制备的驱动器. 其中, 由湿度引起的加捻
纱线驱动器已得到广泛研究. 例如, 经过氧等离子体处 理的渐变扭力碳纳米纱线具有和水及水分子反应的高 可逆性, 进而制备快速响应大收缩和旋转的执行器. 另 外，在加捻的疏水性碳纳米纱线中添加吸水材料也是 一种常用的研究湿度执行器的方式 ${ }^{[32]}$. Kim等人 ${ }^{[33]}$ 报 道了一种复合纱线基的执行器，该执行器由卷曲的碳 纳米管主体和亲水性的聚二烯丙基二甲基氯化铵构成. 通过改变执行器周围的相对湿度大小或与水接触可以 实现驱动. 该湿度执行器可提供高达 $78 \%$ 的拉伸冲程, 大重力功容 $\left(2.17 \mathrm{~kJ} / \mathrm{kg}\right.$ )和大体积功容 $\left(1.8 \mathrm{MJ} / \mathrm{m}^{3}\right)$. 值 得一提的是, 基于天然纤维的湿度执行器, 提供的输出 应变压力和驱动能力比动物骨骼肌和许多由合成材料 制成的执行器要高几个数量级 ${ }^{[16]}$.

气动执行器是可穿戴执行器的一种常见形式，可 通过气压变化来改变材料的形状. 针织纺织品、机织 纺织品和中间的薄安全气囊已集成在一起，用于制备 执行器 ${ }^{[34]}$. 当中间气囊充气时, 会产生一系列多个角度 的自由活动. 但这一类驱动器也具有体积较大、需要 外部压缩机设备、对材料密闭性要求高等劣势.

由于其他柔性执行器(如电执行器、气动执行器 等)均需要额外设备等的限制或极慢的响应或极慢的 恢复速度(如离子执行器等), 热驱动执行器可以从环境 中获取热源和快速的响应及恢复等，使其开发逐渐成 为织物电子领域研究的重点. 此外, 织物执行器领域仍 然面临着一些挑战：柔性执行器产生的力和运动很小; 驱动面临着可逆性问题; 驱动是不可精确控制的.

\section{3 织物显示器}

传统的显示器笨重且材质较硬，阻碍了可穿戴电 子产品的发展. 以纺织品为基础的显示器有望改变可 穿戴电子产品和时装工业 ${ }^{[35 \sim 0]}$. 近年来, 有机电致发光 装置的研究得到蓬勃发展, 例如有机发光二极管 (OLED)和聚合物发光电化学电池(PLEC). 另外, 无机 发光二极管可直接与织物相连，令可穿戴的纺织品显 示器成为可能 ${ }^{[41 ~ 49]}$. Choi等人 ${ }^{[39]}$ 利用简单、低成本和 低温的方法制备了一种高效能的织物OLEDs. 该织物 OLEDs可在半径为 $3.5 \mathrm{~mm}$ 时承受高达 $4.3 \%$ 的拉伸应变 (图1(a)). Kim等人 ${ }^{[42]}$ 也开发了基于织物的OLED装置 (图1(b)). OLED器件表面通过插人多层阻隔膜阻隔环 境中的水分和氧气等. 即使在环境空气中工作 $3500 \mathrm{~h}$ 后, 所得的双面封装OLED器件也可以保持几乎不变的 性能. 此外, PLEC也可以在粗糙的表面上制备. 如图 1 
(a)

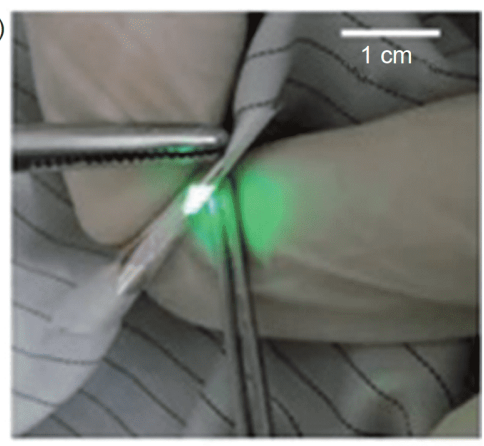

(c)
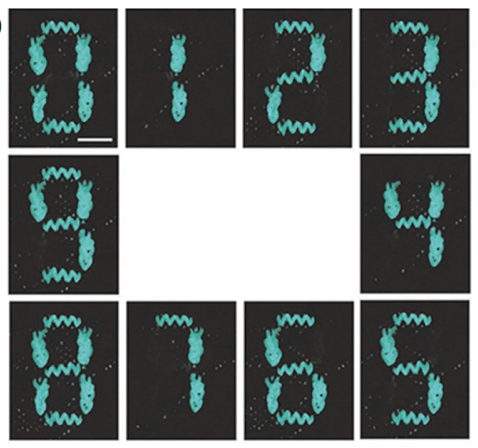

(b)

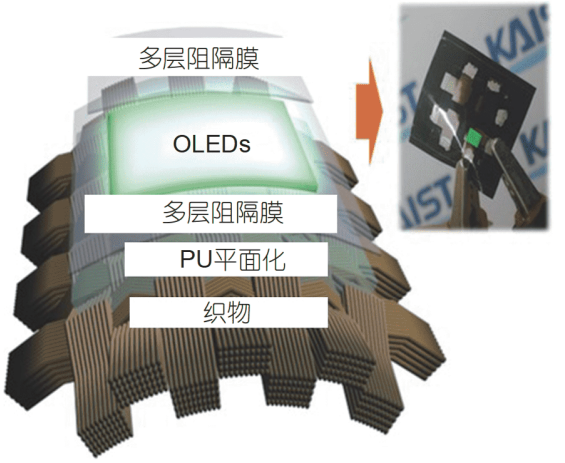

(d)

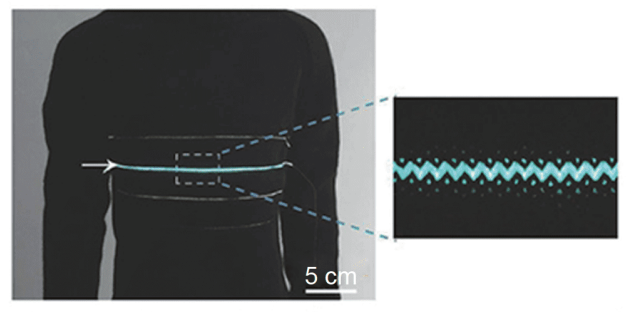

图 1 (网络版彩色)织物基显示器. (a) 弯曲状态下的发光装置 ${ }^{[39]}$; (b) OLED的结构示意图 ${ }^{[42]}$; (c) 纤维型PLEC的结构示意图 ${ }^{[49]}$; (d) 由发光纤维 织成的服装 ${ }^{[50]}$

Figure 1 (Color online) Fabric-based display. (a) Images of a fabric display under bending tests ${ }^{[39]}$; (b) schematic diagram of OLED ${ }^{[42]}$; (c) schematic of the structure of a flexible fibre-shaped PLEC ${ }^{[49]} ;(d)$ an electroluminescent textile ${ }^{[50]}$

(c)所示, Peng研究组 ${ }^{[49]}$ 开发了一种可调颜色、可编织 的PLEC，提供了多种可调的颜色，并显示出可织人智 能织物的潜力. 通过一步挤压的工艺, Peng研究组 ${ }^{[50]}$ 从 连续的电致发光纤维开发出一种新颖的发光装置. 所 制得的发光装置具有灵活、可拉伸、可弯曲、透气的 优点, 可用于计算机通信, 并适合于任意曲率的皮肤且 能通过织造技术嵌人服装内(图1(d)). 尽管基于纤维的 织物显示器取得了重大突破, 但依然存在一些问题有 待解决, 包括: 纤维上电子空穴的不均匀分布导致纤维 基-发光材料性能比平面基的产品差、操作稳定性 差、使用寿命较短 (由于暴露于湿气和氧气，少于 $100 \mathrm{~h}$ )、电子系统中输人/输出端的高分辨率全彩LED 阵列的制备技术仍然受到限制、纤维基材的显示装置 与其他可穿戴的电子器件整合难度大等.

\section{4 织物天线}

天线是万物互联物联网中不可或缺的重要组成部 分. 可穿戴天线采用柔软的纺织品作为介质基板, 通过 加人导电纱线, 从而可以穿戴在人体上 ${ }^{[51]}$, 具有薄、轻 便、坚固的特性, 且易于集成到柔性电路中等特点. 天
线的空间几何结构会受身体运动的影响，影响其性能 和稳定性 ${ }^{[52]}$. 因此, 织物天线应是特别设计的, 例如, 采用较宽的频带带宽以避免遭受机械变形引起的频率 失谐. 目前, 实现天线微型化、结构紧凑且可弯曲变形 是目前的主要研究方向. 此外, 减小天线对人体的辐射, 保证人体的安全性也是当前织物天线的研究重点.

\section{5 产能和储能器}

织物发电机系统 ${ }^{[53 ~ 67]}$ 可以根据热电、光电、压 电、摩擦发电、以及它们之间杂化等机理收集人类活 动和周边环境中的能量. 其中, 将热能和光能转化为电 能最常见的机理主要包括热电效应和光伏效应. Zeng 等人 ${ }^{[56]}$ 将还原氧化石墨烯应用于三维打印的聚二甲 基硅氧烷网格上制备出柔性的热电发电机. 在环境温 差小于 $15^{\circ} \mathrm{C}$ 时, 该演示的腕带式柔性热电发电机的输 出功率密度为 $4.19 \mu \mathrm{W} / \mathrm{g}$. Zhang等人 ${ }^{[57]}$ 建立了一套可 预测三维长丝基热电发电机的输出性能的理论模型. 该三维模型由一维的长丝基热电发电机阵列组成. Chai等人 ${ }^{[68]}$ 开发了一个全固态可调节的织物发电系 统, 该系统可以同时收集和存储太阳能. 这个织物发电 
系统可以在收集 $1.7 \mathrm{~s}$ 太阳能后输出 $1.2 \mathrm{~V}$ 的电压，在放 电电流密度为 $0.1 \mathrm{~mA}$ 的情况下可在 $78 \mathrm{~s}$ 内完全放电. 这些热电发电器件和光电发电器件具有的独特优势 是, 它们不需要任何的移动和变形; 但同样也存在着明 显的缺点, 例如效率较低和受环境影响较大等 ${ }^{[65]}$. 通 过压电、摩擦电、电磁感应、静电感应等机理将机械 能转化为电能的原理则受环境的影响较小. 但基于机 械能转换的发电机系统的结构也变得相对复杂, 因为 部件之间需要存在相对移动或者变形. 由于织物多级 结构的存在, 织物基发电机则更为复杂. 其中, 压电发 电机基于内部晶体形成的不同极性分布，产生感应电 荷. 压电发电机可以封装于比较紧凑的结构中, 如功 能性纤维 ${ }^{[69,70]}$, 有助于发电机的小型化和集成化. 然 而, 由于柔软的织物难以为压电单元提供足够的压缩 或者拉伸变形, 这类织物压电发电机的电输出一般非 常低(峰值电压为几十毫伏到几伏). 与压电发电机相 比, 摩擦发电机结合摩擦起电和静电感应原理将不规 则的机械能转化为电能, 可以在低应力水平和低频率 下工作，同时也具备更高的表面电荷密度和更高的输 出 ${ }^{[71,72]}$. 从 2012 年Wang研究组 ${ }^{[73]}$ 提出纳米摩擦发电机 以来, 摩擦发电机技术发展迅速, 输出性能方面有极大 的提升. 尽管织物摩擦发电机可以获得较高的瞬时功 率, 但当它们往电容里充电作为储备能源时, 它们的 充电性能依然较低无法满足常见电子设备的需求. 因 为这类发电机主要以脉冲的形式输出电能, 其峰值维 持的时间很短，一般为几毫秒到几十毫秒. Tao研究 组 ${ }^{[65]}$ 对不同种类的可穿戴式发电机, 包括电磁发电 机、压电发电机、摩擦发电机、热电发电机、太阳能 发电机和它们之间的杂化发电机进行了综合对比，表 明电磁发电机可产生最高的平均输出功率 (达 $32 \mathrm{~mW}$ ). 但截至目前, 电磁发电机很少可以封装为织物结构. 因 为高效电磁发电机需要磁铁和导电线圈之间存在快速 的相对移动, 从而形成极高的磁通量变化率. 同时, 现 有的磁铁和导电线圈的密度大, 且器件中磁铁和导电 线圈的用量也较大, 导致难以生产轻量化的小体积器 件. 另外, 织物杂化发电机也是提高电输出的有效手 段 ${ }^{[65,70]}$.

在实际应用中，电能常常被存储起来作为储备能 源, 为织物电子器件供电. 最为常见的储能器是超级电 容器和电池. 超级电容器在功率密度、快速充放电和 长周期稳定性方面具有优异的表现，而电池则在能量 密度、高工作电压和低自放电方面具有优势. 超级电
容器和电池都是有限寿命和有限容量的, 频繁的充电 和更换器件会带来极大的不便利. 为解决该问题, 集成 系统，包括能源收集或电能产生装置、电源管理和储 能器等, 可成为一种目前能源供给的替代方案. 随着集 成技术的发展, 一体式超级电容器也逐渐出现, 其更容 易被封装于三维的电子器件中. 一体式超级电容器同 样拥有较高能量密度 $\left(2.12 \mathrm{mWh} / \mathrm{cm}^{3}\right)$ 和功率密度 $(320$ $\mathrm{mWh} / \mathrm{cm}^{3}$ ), 经历 $431 \mathrm{~h}$ 的连续操作后, 超级电容器的电 容没有衰减 ${ }^{[74]}$. Tao研究组 ${ }^{[65,75,76]}$ 和Kim 等人 ${ }^{[33]}$ 最近总 结了织物存储器件的进展. 这些结果表明, 尽管近年来 在能量和能量密度方面取得了较大的进展, 但在容 量、耐用性、延展性、稳定性、可扩展性、安全性和 设备集成性能方面离实际应用依然存在差距.

未来织物电子系统会用System-on-fabric形式以实 现智能化. 除了以上所述柔性器件, 织物电子系统还必 须包括信号调制模块、计算器、存储和无线通讯模块 等微电子器件. 其中, 信号调制模块从织物传感器获得 的数据转换为控制器识别的信号. 例如, 对于电阻型的 器件, 通常先将电阻变化转换为电压信号 ${ }^{[77]}$. 微控制器 处理数据后，通过无线模块进行信号/数据交换. 目前, 织物电子系统在电子电路研究中存在的问题包括: (1) 缺乏能将纺织设备的输出信号转换为数模转换器, 可 以识别电压信号的标准电路; (2) 缺少适合织物电子系 统的信号调节电路; (3) 所形成的电路体积大, 刚性大 并且容易断裂; (4) 缺少从尺寸、功能和能耗等方面合 理配置控制器、数模转换和无线模块等的标准.

\section{2 科学问题}

织物电子系统与传统的微电子系统或电子系统在 材料、结构、集成等方面存在巨大的差异. 同时, 对织 物电子在生物相容性、水洗性、舒适性方面也提出更 高的要求. 围绕可靠性、交叉兼容性和标准, 在设备适 用性、材料可用性和成本等方面限制了现在大部分电 子产品的商业化. 如何在保留织物电子器件和系统的 功能性与可靠性的同时, 还具备织物优异的可穿戴性 是目前织物电子领域发展的目标和瓶颈所在. 此外, 织 物电子器件和系统具有多尺度 (从小纤维到三维大面 积结构)、系统集成的多物理场、复杂多变的应用环 境等特点, 使得织物电子器件或系统研发的综合分析 变得更为困难. 目前, 织物电子器件和系统在研发过程 中存在的一些亟须探索和解决的科学问题, 包括:

（1）建立柔性功能材料和结构的测量和表征系统. 
织物电子系统包括传感技术、数据传输和存储、数据 显示、控制、能源供给技术、连接技术等，其使用的 材料包括金属、非金属、聚合物等. 织物结构由低维 度的材料通过编织技术构成复杂的三维结构, 其尺度 跨度从纳米、微米到米或千米, 从小纤维到大面积织 物. 这些柔性材料/结构具备了良好的生物相容性, 但 基于这些材料/结构建立的传感技术缺乏良好的重复性 和稳定性. 同时, 它们还需要满足可水洗、可承受大变 形并能恢复至初始状态和适应可变环境(如不同的温 度、湿度、 $\mathrm{pH}$ 等). 如何对这些功能材料和结构进行测 量和综合表征是当前亟待解决的重大问题之一.

(2) 建立适用于织物电子器件的机理和数学模型. 织物电子器件以纤维集合体为载体或者纤维材料本身 具备电子功能, 其工作机理与其纺织结构的力-电特性 密切相关. 织物器件中的结构设计、材料选择、功能 材料的界面效应及变形等对电场分布、载流子流动和 器件性能等有较大的影响. 织物结构复杂, 它是纤维的 集合体, 且纤维之间可相对移动和存在摩擦. 此外, 织 物器件的硬度和模量远低于传统电子器件和微机电系 统器件, 而且织物电子系统各部件的尺度差异可跨越 几个数量级. 因此, 传统硬质电子器件的模型和机理难 以直接解释织物电子器件, 织物电子领域需针对性地 建立织物电子器件工作机理和数学模型, 用以指导 研发.

(3) 建立织物电子器件的多物理场耦合效应分析 及其对性能的影响. 在加工和使用过程中, 织物电子器 件经常处于多物理场共同作用下. 例如, 织物电子器件 应能承受机洗和烘干的纺织品保养的要求, 在这个过 程中, 织物电子器件需要承受外力作用, 同时受到湿度 和温度变化的影响. 在多物理场作用下, 除电子性能方 面的影响外, 其所导致的界面分离是阻碍织物电子器 件发展的另一个因素. 界面分离可以直接导致载流子 无法从一种材料运动到另一种材料, 进而导致界面处 电阻增大, 甚至器件失效. 此外, 织物电子器件也会在 多个物理场共同作用的条件下工作, 例如织物热电发 电机在工作过程中同时受到热场和电场的作用 ${ }^{[57]}$.

(4) 建立织物电子器件和系统的评价系统. 织物电 子结合了传统电子器件和纺织品的功能和特性, 是两 个领域的综合产物, 因此织物电子的要求有别于传统 电子器件和纺织品. 织物电子器件和系统的评价系统 可以分为两个方面: 电子性能和织物性能. 在织物性能 方面, 织物电子应具有可穿戴性能, 因此它的评价系统
具有服用性能，包括尺寸稳定性、透气、外观和手感 等. 织物电子在使用过程需要经过多次的洗涤和穿戴, 穿戴过程中会有不同的弯曲和拉伸程度, 因此织物电 子器件需严格测试其耐用性, 包括耐水洗、耐汗渍、 耐弯折、耐磨性等. 目前, 不同国家和地区对织物电子 评价系统的发展仍然处于萌芽阶段. 欧洲标准化委员 会的248技术委员会(CEN/TC 248 WG 31)于2011年发 布了两个标准, 仅涉及导电纺织品和包含相变材料的 纺织品 ${ }^{[78]}$. 目前, 电子纺织品标准尚不全面, 仅涵盖部 分产品.

(5) 织物电子的生物、信息安全问题. 随着织物电 子的发展, 安全性问题日益受到关注. 织物电子器件和 组件使用纳米材料、半导体和金属材料. 这些材料需 要考虑其对人体和环境的影响, 进行综合评估. 例如, 纳米粒子具有尺寸小、比表面积大、表面态丰富、化 学活性高等性质, 表现出很强的生物毒性, 会导致组织 器官生长发育迟缓、细胞分裂异常、细胞凋亡 ${ }^{[79,80]}$ 等. 能量转换和存储器中通常会引入电解质, 应避免其 泄漏和建立完善的回收处理机制. 织物电子应满足日 常长期贴肤穿着使用需求, 因此必须建立预防漏电和 过热的保护机制, 避免汗液等的侵蚀和因过热引起皮 肤梁伤等. 织物电子系统收集了大量的个人生理数 据、环境数据、地理位置和生活习惯等信息, 这些信 息的泄漏可能导致不当的使用、欺计和一系列社会 问题.

\section{3 核心技术}

\section{1 功能材料和制备工艺}

新型功能材料主要包括新型柔性本征导电材料、 石墨烯及其衍生物、有机-无机复合材料等. 其中, 新型 的柔性本征导电材料主要分为金属纤维、本征导电聚 合物、碳基材料及其衍生物. 在本征导电材料中, 金属 因其具有出色的导电率是最早用于发展柔性电子器件 的导电材料. 金属和合金可通过牵拉技术制成导电纤 维或长丝 ${ }^{[81]}$. 然而, 金属纤维和长丝因其高刚度、高硬 度、高密度、低柔韧性等性能限制了其应用范围. 随 着纳米尺度涂层技术的发展, 纤维表面通过涂覆金属 纳米线或纳米棒形成新型的导电纤维 ${ }^{[82]}$, 但其均匀度 和稳定性还存在挑战. 与金属不同, 导电高聚物本身具 有很好的柔性, 并且具有较好的热稳定性、环境稳定 性、导电性、易加工性等 ${ }^{[83]}$. 其中, 最常见的导电高聚 
物包括聚苯胺(PANI)、聚吡咯(PPy)和聚 $(3,4$-乙烯二氧 噻吩)-聚苯乙烯磺酸(PEDOT:PSS). 然而, 与金属材料 相比, 本征导电高聚物的导电率比金属材料差 $4 \sim 5$ 个数 量级, 仅处于半导体阶段. 在实际使用中, 该材料常需 要混合其他高导电率材料, 如石墨烯、碳纳米管、碳 纤维等. 碳基材料拥有优良的拉伸性能、导电性能和 较大比表面积, 是织物电子领域常用材料之一 ${ }^{[84,85]}$. 由 于小尺度的碳材料在使用过程中可能进人人体, 并对 人体可造成严重伤害, 因此需要谨慎使用和进行综合 评估, 尤其要注重用户安全和环境保护.

常用的制备工艺主要包括丝网印刷、喷墨打印、 气溶胶喷墨打印、涂层技术、物理气相沉积技术、 镀、化学气相沉积技术、原位聚合、碳化等.

(1) 丝网印刷通过刮板将颜料挤压出丝网, 在基底 上印刷出图案 ${ }^{[86,87]}$, 可用于大规模生产织物电子和重 复生产高纵横比的图案. 但丝网印刷需要高黏度的颜 料, 会降低纺织品的透气性和柔性, 且所得图案分辨率 较低.

（2）喷墨打印通过将一定量的墨水挤出糟, 并穿过 喷嘴打印到基底上, 易于设计图案. 常用墨水包括金属 性墨水、氧化石墨烯墨水、本征导电聚合物和碳纳米 管墨水等 ${ }^{[88]}$. 多孔的基底容易导致打印的图案出现断 层, 喷头容易被堵住和低黏度墨水难以打印出高电阻 的图案等是目前喷墨打印常见的问题.

（3）气溶胶喷墨打印利用空气动力形成高分辨率 的胶态悬浮体. 气溶胶喷墨打印可以采用多种墨水和 基底, 并且不会出现喷头堵塞的现象. 基底可以包括平 整和不平整的材料、二维和三维材料. 气溶胶喷墨打 印的主要缺点在于打印图案的边缘会因其机理有波浪 和微点, 难以形成边缘整齐的图案.

(4) 涂层技术是将半流质的材料涂到纺织品上. 涂 层技术可以直接将非导电纺织品转为导电纺织品, 而 不需要将任何电子器件或电子材料在纺织品生产过程 中嵌人纺织品而得到功能性纺织品. 涂层技术的重点 是涂层和基底的黏附, 其主要受到基底的浸润和涂层 材料的黏度所影响.

(5) 物理气相沉积是一个蒸涂过程. 在该过程中, 材料转换成不同的形态并形成沉积层到纺织品表面. 物理气相沉积可以分为喷镀、真空蒸镀、气相沉积和 离子电镀, 其中喷镀和真空蒸镀比较常见.

(6) 镀可以应用于不同的纺织品, 一般可以分为电 镀和化学镀. 化学镀是发生在水溶液的化学反应 ${ }^{[89]}$, 可
以将非导电纺织品转变为导电纺织品, 还可以通过不 同的前处理工艺改善镀层和基底的结合力, 如等离子 处理、化学刻蚀等 ${ }^{[90]}$. 电镀则是通过电流驱动金属离 子还原到织物表面. 在电镀过程中, 织物是阴极, 电流 穿过溶液, 沉淀金属到织物表面 ${ }^{[1]}$. 电镀的前提条件是 基底必须是导电的，因此大部分的纺织品并不适合用 此方法来开发织物电子器件.

(7) 化学气相沉积主要是通过化学还原反应沉积 气态化学前体到织物表面. 反应可以由不同的能量引 起, 包括热能、离子或者光. 在化学气相沉积槽中, 不 同的引发剂和单体传送至槽中, 之后结合到织物表面 进行化学反应. 目前, 此法应用到织物的研究仍然有限.

（8）原位聚合是指气体或者液体的基体发生聚合 反应. 纺织品浸人或置放于含有单体的气体或者溶液 中, 加人引发剂从而引发聚合反应. 原位聚合的好处在 于不会对基体的性能产生较大的影响, 并且可以进行 大规模的生产. 但原位聚合反应的缺点包括无法实时 监控反应过程, 其效能的重复性较低, 部分原位聚合溶 液因含有酸或碱对基底等造成损害 ${ }^{[92]}$.

（9）碳化是一个热处理过程, 把纺织品在惰性环境 中加热. 碳化将改变部分或全部纤维的化学结构和物 理性能, 纺织品的性能会被损害. 例如, 碳化后的纤维 尺寸会减小, 表面面积减少 $49.2 \%$, 重量减少 $84.7 \%{ }^{[93]}$. 此外, 该工艺会对环境带来污染, 生产过程中会产生大 量有毒气体, 包括氨气和㲵化氢 ${ }^{[94]}$.

\section{2 织物器件的结构和功能理论及表征方法}

根据不同的维度, 织物器件的结构可以分为一维 纤维或纤维基器件和织物基器件. 根据结构不同, 一维 纤维或纤维基器件可以分为两类, 包括由本征导电材 料(如金属、本征导电高聚物和碳基材料)直接制成的 导电纤维和通过混纺、牵拉或涂覆等方法制成的具有 皮芯结构的器件. 如图2(a)所示, 多功能、多组分的电 子纤维已经在研究中通过汼拉技术制成 ${ }^{[95]}$. 首先, 将高 聚物、低熔点金属和微型芯片装备到一个网孔结构中, 之后对网孔结构加热到金属和高聚物的熔点温度, 经 过机械牵拉从而得到多功能、多组分的电子纤维. 这 种方法解决了微/纳米制造中准备多功能纤维费用高昂 的问题. 但为了保持牵拉过程中稳定的结构, 熔点温 度、黏度和热膨胀系数必须高度匹配. 这些要求减少 了这种纤维中组分的可选择性. 在纤维、纱线或者织 物表面涂覆导电材料也是一种可以批量生产织物电子 
(a)

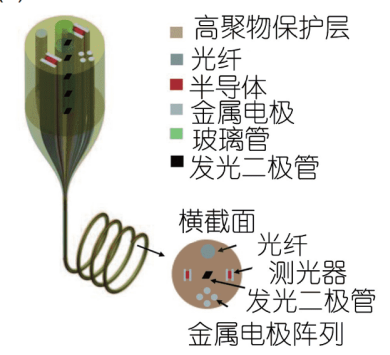

(b)

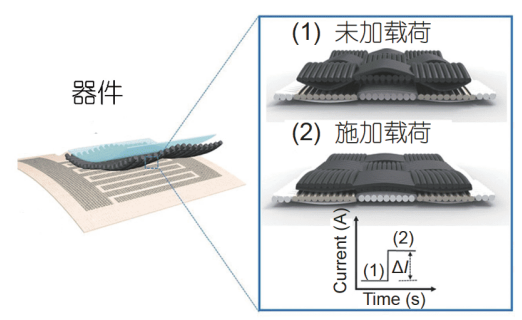

(c)

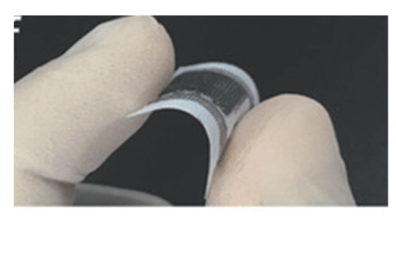

(f)

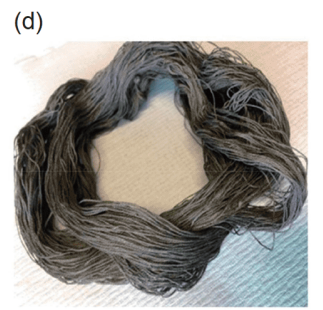

(e)

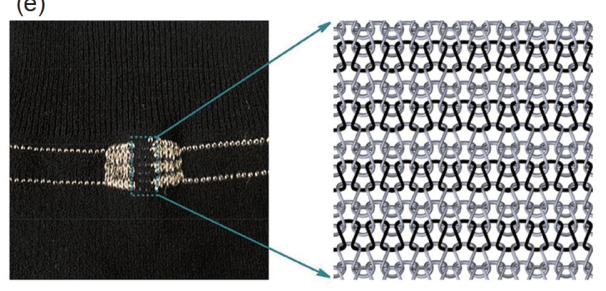

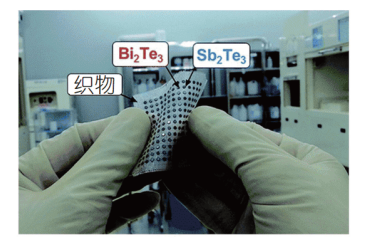

图 2 (网络版彩色)织物电子的功能性器件. (a) 热牵拉多功能不同组分的纤维示意图 ${ }^{[95]}$. (b) CNT涂覆的棉织物和镍涂覆的涤纶织物构成的压

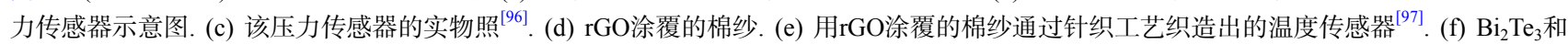
$\mathrm{Sb}_{2} \mathrm{Te}_{3}$ 薄片被嵌人到玻璃纤维机织物中构成的柔性热电器件 ${ }^{[98]}$

Figure 2 (Color online) Functional components of fabric electronics. (a) Schematic diagram of the thermal drawing of multimaterial fibers with different embedded materials and functionalities ${ }^{[95]}$. (b) Schematic illustration of the fabrication procedure of textile pressure sensors ${ }^{[97]}$. (c) A image showing the bendability of the textile sensor. (d) Hank of rGO-dyed (coated) cotton yarn. (e) Knitted temperature sensor with graphene-coated yarn ${ }^{[96]}$. (f) Photograph of $\mathrm{Bi}_{2} \mathrm{Te}_{3}$ and $\mathrm{Sb}_{2} \mathrm{Te}_{3}$ dots on a glass fabric ${ }^{[98]}$

器件的方法，通过这种方法得到的织物电子器件基本 保持了原有纤维/织物的柔性. 如图2(d), (e)所示, 通过 采用成批纱线染色技术，得到了还原氧化石墨烯(rGO) 涂覆的棉纱，并用该棉纱通过针织技术织造了温度传 感器 ${ }^{[97]}$. 织物基器件也可以分为两类: 一维纤维或纤维 基器件通过纺织织造工艺(如机织、针织等)直接加工 得到的器件和将电子元件嵌人到织物中得到的器件. 如图2(b), (c)所示, 利用镍涂层的涤纶织物和碳纳米管 (CNT)浸涂的棉织物构成了面面接触的压力传感器 ${ }^{[96]}$. 这种先得到功能性纱线，后通过纺织工艺直接制造织 物电子器件的方法, 要求制造过程的张力和摩擦等因 素不损伤功能性纱线的性能. 此外, 也可将已经制备好 的电子元件直接嵌人织物中, 各个元件之间通过导线 连接, 从而在整体上保留了织物的柔性. 比如, $\mathrm{Bi}_{2} \mathrm{Te}_{3}$ 和 $\mathrm{Sb}_{2} \mathrm{Te}_{3}$ 薄片被嵌人到玻璃纤维机织物中, 从而得到了 一款柔性热电发电机, 如图2(f)所示 ${ }^{[98]}$. 但由于这两种 半导体材料制成的薄片硬度大，在穿戴过程中的舒适 性不足.

\section{3 纺织器件和电子器件的异质集成设备和工艺}

通过机织、针织、刺绣等纺织工艺可以直接开发 织物基电路板, 然后连接织物电路板和电子器件. 这些
工艺可以保留电子元件的电性能和织物的柔软性. 本 研究组 ${ }^{[99]}$ 采用两组正交垂直的纱线生产机织织物电路 板, 并用该电路板来连接各种电子元件(例如温度传感 器, 图3(a)). 此外, 还用针织的方法把涂覆聚氨酯的铜 纤维和弹性长丝编织成三维可变形的针织物电路 板 ${ }^{[100]}$, 如图3(b)所示, 实现传感器与织物电路板上的导 线有机械和电气的两重连接. Ismar等人 ${ }^{[101]}$ 也通过刺绣 的方法把导电纱嵌人织物中形成导电通路, 然后用焊 接的方法连接LED和导电纱线, 所形成的织物基电路 及LED如图3(c)所示. 通过绣花布线形成织物电子电路 比机织和针织更简单, 但是嵌人绣花织物表面的导电 线可能会导致电气短路.

除了上述织物电路的集成方法, 织物电子系统的 另一个难点是织物和电子之间的异质集成，即织物和 电子的互连. 由于织物电子系统中芯片等的连接面积 非常小, 常用的方法连接有焊接和导电胶黏剂. 但这些 方法也存在挑战, 如LED元件与织物上低熔点焊点之 间的连接失效(图3(d) $)^{[102]}$. 柔性导电织物和刚性连接 器或电路金属线之间进行电连接 ${ }^{[104 ~ 106]}$. Tao研究组 ${ }^{[103]}$ 建立了一种利用一个螺旋结构实现织物电子与针织电 路板上导电线路的柔性连接方法(图3(e)). 这种柔性连 接特性使织物电子在沿轴向拉伸时保持稳定, 但不如 


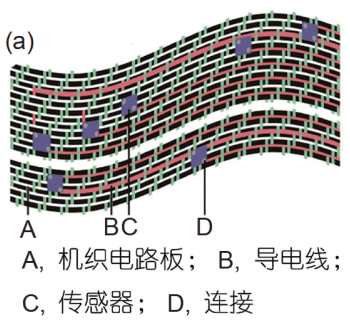

(d)

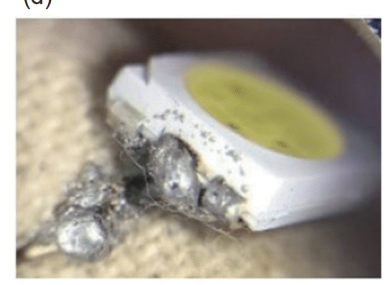

(b)

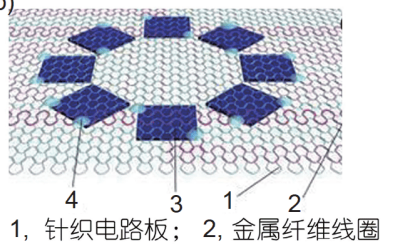

3 , 传感器; 4 , 连接

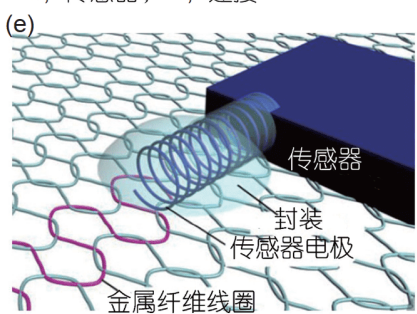

(c)
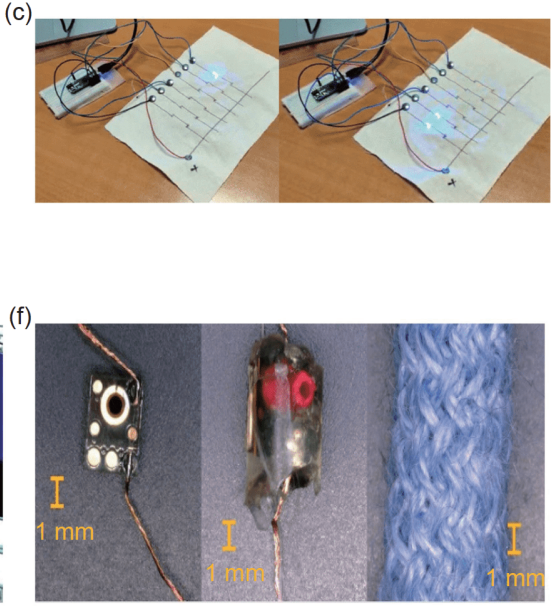

图 3 (网络版彩色)织物电子的异质系统集成. (a) 机织电路板及传感器连接 ${ }^{[99]}$; (b) 针织电路板和传感器连接 ${ }^{[100]}$; (c) 刺绣电路和LED连接 ${ }^{[101]}$; (d) 织物和芯片的焊接失效模式 ${ }^{[102]}$; (e) 机械加持连接 ${ }^{[100]}$; (f) 铜线连接芯片, 封装后的芯片和铜线包缠到纱线里 ${ }^{[103]}$

Figure 3 (Color online) Fabric electronics architecture and system integration. (a) Structure of the fabric sensing network ${ }^{[99]}$; (b) fabric sensing network integrated with sensors ${ }^{[100]}$; (c) image of the embroidered circuit board with six parallelly connected $\operatorname{LEDs}^{[101]}$; (d) types of joint failure ${ }^{[102]}$; (e) helical connection from the sensor electrode to conductive track of the knitted circuit board ${ }^{[100]}$; (f) fabrication procedures of acoustic sensing yarn ${ }^{[103]}$

刚性连接稳定, 在大变形时也可能发生断裂. 导电胶黏 剂由金属或导电粒子和聚合物黏合剂组成, 可用来连 接电子元件和柔性电路. 使用导电胶形成电连接的主 要优点是操作简单和需要适当的封装层(图3(f)), 而人 体湿度和环境温度会影响导电胶的电气连接质 量 ${ }^{[107]}$ 等.

\section{4 纺织电子器件及系统的工程设计软件}

纺织系统的工程设计软件一般指计算机辅助设计 (CAD), 指利用计算机强大的计算功能、图形设备的处 理能力, 实现织物的花型结构设计、仿真和展示 ${ }^{[108]}$. 目前, 针对常规织物设计的 CAD系统已经有较完善的 功能, 如织物的花型数据结构、花型编辑、成形工 艺、线圈模拟等. 但是针对功能性织物电子系统的设 计软件还处于婴儿期. 例如, 目前, Rathnayake ${ }^{[109]}$ 研发 出电子纱线, 即把电子元件集成到纱线中, 既保证了纱 线的柔软等服用特性, 同时也使纱线具有多种功能. 该 电子纱线可以测量人体温度、测试振动等, 但需要单 独的设计模块来确定纱线中电子元件在织物中的结构 和定位等. 纺织电子系统设计软件未来应考虑的方向 有: 首先, 在现有的工程设计软件上增加功能材料数据 库和设计模块. 新增设计模块应综合考虑普通纱线、 电子纱线、织物结构、器件结构, 及器件电子、力 学、热学性能等. 其次, 开发能精确放置电子元件的软 件模块. 最后, 该软件系统还包括电子系统设计、服装
载体设计、人体工学和效果展示等功能.

\section{5 特种光纤器件、光电器件、系统微型化}

光纤器件在压力、温度、气体和液体的测量精 度、可靠性、抗干扰性等方面都比电子织物器件优越. 特种高分子光纤器件从原理上和实验室都作出了明确 的证明. 光纤、发光二极管和激光二极管等的尺度已 经很小, 可以直接集成到纱线和织物中. 目前限制其在 可穿戴系统应用的主要障碍是测量系统尺寸太大以及 高额的设备费用. 此外, 由于测量设备有超高的输出稳 定性和环境稳定性等要求, 导致其微型化进展缓慢. 最 为重要的是, 国内高端的光纤器件目前主要从美国、 欧洲国家等进口, 如美国的Micron Optics公司. 我国在 光纤器件方面与世界先进水平之间还存在较大的差距, 需要提高重视、加大投人及立项研究.

\section{6 纺织电子器件及系统质量保证体系}

目前, 纺织电子器件及系统的质量保证体系还处 于接近空白状态. 传统纺织行业的测试标准不涉及电 子器件特性, 而现代电子行业的规范未涉及服用特征 指标. 因此, 目前的规范未能适用于织物电子器件和系 统. 广州纤维产品检测研究院在所承担的项目“智能可 穿戴纺织品的应用研究及安全评价体系建立”里提出 智能产品的安全性应包括: 化学危险物质、电池和信 息安全、电磁辐射及环境安全性等 ${ }^{[110]}$. 但该安全性评 
价体系目前并未普及. 织物电子领域的专家应建立织 物电子行业的细分和可量化的国内/国际标准. 此外, 这些标准还应包含生物兼容性、材料安全性等关键安 全指标.

\section{4 应用展望}

\section{1 医疗健康}

随着社会人口老龄化的加剧, 对医疗服务的需求 和医疗行业的压力不断增加. 通过发展织物电子, 将电 子器件或功能嵌人到传统的纺织品中, 有望打破传统 医疗行业在地域和时间方面的限制，舒缓医疗行业的 压力. 目前在医疗方面, 织物电子主要应用在柔性测量 技术中. 通过监测人体生理参数, 如心电、脑电、运动 特征等, 去判断人体当前的生理状态 ${ }^{[111,112]}$, 并建立数 学模型对跌倒、损伤、突发性疾病或其他高风险状态 的发生提供监测或预警信号. 除了远程监测, 远程治疗 也成为织物电子的一大潜力发展趋势. 根据远程监测 数据或反馈, 远程控制织物电子器件进行远程治疗, 如 物理治疗、中医按摩等. 因其具有类衣物的贴合性和 随身性, 织物电子系统可以将部分治疗融人日常生活 中, 提供更贴合生活环境的监测和治疗, 增加其疗效, 尤其是在心理治疗方面.

\section{2 运动监测和辅助训练}

智能运动服装在提供基本保护的同时还可以获取 人体和周边环境的信息, 建立更进一步保护机制, 如避 免因过度运动和不当运动造成的损伤. 例如, 将不同的 电子器件融人运动服装中, 实时提取人体生理信息、 周边环境信息、运动姿势等并进行分析和判断, 以提 高运动质量和避免运动伤害等. 这些智能设备也可以 应用到运动员的日常训练中. 例如, 在肘关节弯曲的等 距收缩训练时, 上臂围度会随着肘部肌肉的收缩而增 加. Tao研究组 ${ }^{[13]}$ 在其开发的织物应变传感器基础上 开发了一套无线、柔性可穿戴的上臂原位测量系统. 该系统通过监测上臂围度变化和变化率等建立了其与 肌肉收缩/放松的定量关系, 有望用于举重运动员的日 常力量训练.

\section{3 智能防护}

智能织物电子系统一般包含感知、执行、传输和 控制等功能模块, 能接收环境和使用者的生理信息并
对其进行分析和判断, 获得使用者自身的健康状况和 所处环境的风险. 嵌人不同的电子系统可使织物电子 系统具备不同的防护功能. 例如, 嵌人气体传感器可以 检测环境中的有毒害物质, 预防化学危害; 嵌人定位传 感器可检测服用者的地理位置; 嵌人温湿度传感器可 以检测环境中的温度湿度等. 当发现高风险情况, 及时 对用户提供预警信号或启动保护方案. 在意外情况发 生后, 织物电子系统还能根据环境和人体生理状态发 出求救信号和定位信号, 甚至还能提供适当的保护措 施, 避免多次伤害. 例如, Blecha等人 ${ }^{[114]}$ 开发了一套智 能消防防护服系统, 其包含心率检测模块、定位模 块、远红外温度检测模块、传感器模块、报警模块、 服装控制模块和指令控制模块. 该系统完全集成到消 防防护服中, 能实时监测消防队员的心率及运动状态, 并同时监测其周边环境的变化, 如温度、湿度以及是 否存在有毒气体等. 当发现危险状态时, 消防服就会自 动发出声光报警. Reiffenrath等人 ${ }^{[115]}$ 为执法人员等研 究开发了一套轻便的防护服装, 包括心率监测、伤害 评估系统、地理定位模块、无线传输模块及织物天线 等. 该研究还提升了对子弹射击和穿刺的保护. 因此, 这类智能防护服装可以有效提高工作的安全性和降低 伤亡.

\section{4 增强现实/虚拟现实}

互动式织物电子器件具备优异的性能和独特的特 点, 如质轻、灵活、可大变形等, 能为增强现实 $(\mathrm{VR}) /$ 虚 拟现实(AR)应用提供更贴身、更舒适的用户体验. 在 增强现实/虚拟现实系统中, 目前最常用的是织物电子 器件包括柔性执行器、压力传感器、连接器和干电极 等. 最近, Tao研究组 ${ }^{[21]}$ 提出了一种由螺旋复合纱 $($ 聚酰 亚胺 $(\mathrm{PI}) /$ 铜镀层 $(\mathrm{Cu}) /$ 聚二甲基硅氧烷(PDMS))构成的 柔性执行器执行, 如图4(a)所示. 该执行器利用电热效 应进行驱动, 表现出优异的热驱动性能: 在 $-50 \sim 160^{\circ} \mathrm{C}$ 的温度范围内和 $1.2 \mathrm{MPa}$ 张力下可实现达 $20.7 \%$ 的形变. 将该驱动器集成到一个柔性手模型上, 通过加热可以 轻易调整该模型的姿态, 如图4(b)所示. 但由于该驱动 系统的电-热-机械的复杂关系, 该系统暂未能实现精确 的控制. 另外, 运动跟踪和姿势识别是织物增强/虚拟现 实设备的常用功能. 织物电子器件和系统的优异生物 相容性, 使得其在与人体接触方面存在天然优势. $\mathrm{Shu}^{[116]}$ 将柔性织物压力传感器集成到织物鞋垫开发了 一套智能可穿戴系统，该系统可测量足底压力分布和 
(a)

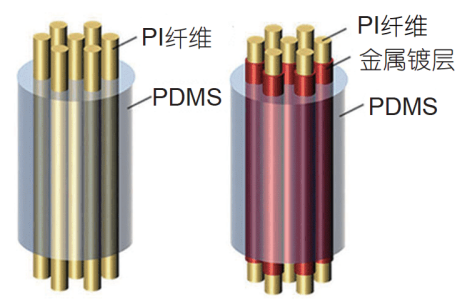

(b)
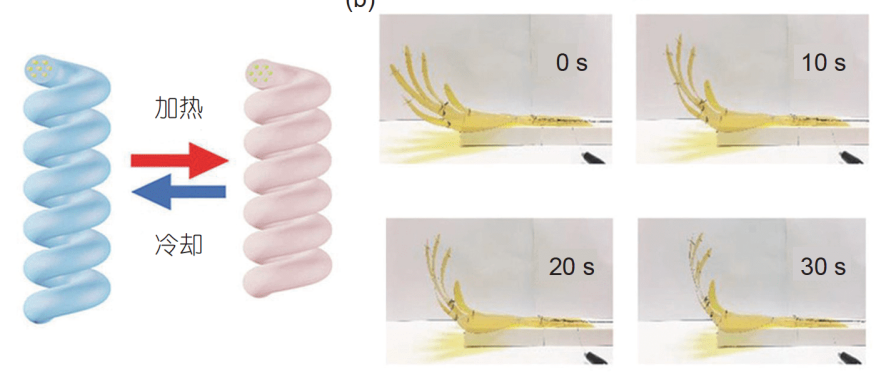

(d)

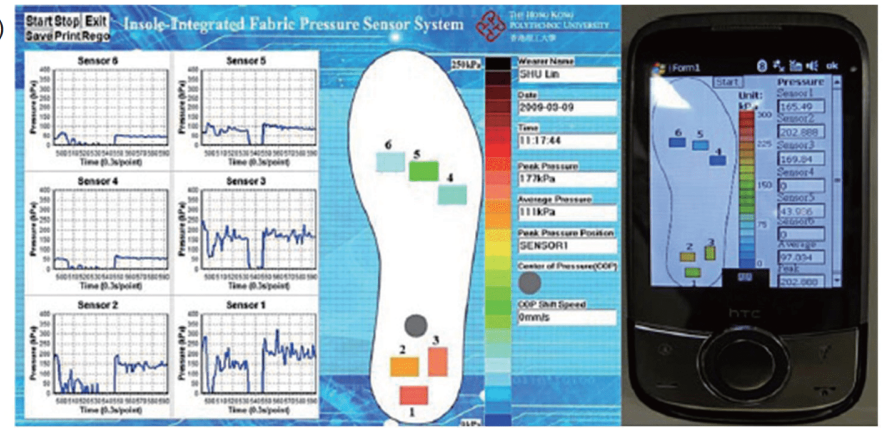

\section{用于虚拟控制的智能手套}

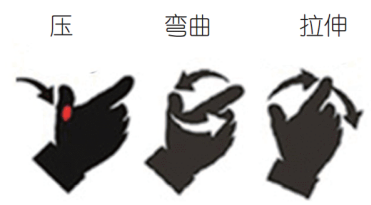

图 4 (网络版彩色)织物电子器件在增强现实(VR)和虚拟现实(AR)的应用. (a) 基于PI纤维的制动器 ${ }^{[21]}$; (b) 制动器驱动机器手 ${ }^{[21]}$; (c) 用于步态 分析的织物系统界面 ${ }^{[116]}$; (d) 用于虚拟现实控制的智能手套 ${ }^{[117]}$

Figure 4 (Color online) Applications of textile electronics in virtual reality (VR)/augmented reality (AR). (a) Structure and components of composite yarn and spring-like thermally/electrothermally powered helical composite yarn actuators ${ }^{[21]}$; (b) robotic hand actuated by helical composite yarn actuators $^{[21]} ;$ (c) plantar pressure data interface ${ }^{[116]} ;$ (d) smart wearable glove for virtual interface control ${ }^{[117]}$

分析使用者步态. 同时，该鞋垫与普通鞋垫相似，使用 者不会产生存有异物感或不适感. Choi等人 ${ }^{[117]}$ 将一种 纤维基压力传感器编织在手套中, 形成一双智能织物 手套. 如图4(d)所示, 该手套可以作为可穿戴的虚拟控 制器. 因为该手套受到外部刺激时, 所测电阻与初始电 阻的比率会随着外力的不同而变化.

Lee等人 ${ }^{[118]}$ 提出利用织物电子(可穿戴式散热器) 进行微气候控制，以增强可穿戴 VR/AR系统的沉浸感. 如图5所示, 该可穿戴式散热器与运动跟踪手套相结合 展示了在虚拟环境中用户可以模拟触摸不同温度的物 体, 比如冰冷的啤酒瓶、软饮料瓶、一杯温暖的绿茶 或热咖啡. 此外, 人体表面生物电位信号, 包括心电 图、眼电、脑电图和肌电图, 也被作为 $V R / A R$ 系统的 输人端来研究. 例如, 利用脑电图解码用户在 VR/AR环 境中的注意力状态 ${ }^{[119]}$ 和评估与增强/虚拟系统相关的 心理-物理效应. 目前, 虽然VR/AR系统还不能完全由 织物电子构成, 但更柔软舒适的织物电子器件已显示 了为用户提供更友好的沉浸式VR/AR体验的巨大潜力.

\section{5 结论与展望}

本文归纳了织物电子器件和系统的发展情况, 总
结了相关科学问题, 并结合在可穿戴领域科研和产业 化过程中的经验和教训, 指出了未来发展的关键技术. 在材料方面, 缺乏安全无毒、电导率可调的、易于结 合又不影响纺织品原有机械性能的、可承受机洗和烘 干的导电和半导体材料. 织物电子传感器件的精度 低、发电器件的输出能量低、器件的稳定性和耐用性 等不足, 难以满足大规模实用需求. 另外, 电子器件的 机理和模型需要拓展, 提高在织物电子器件中的适用 性. 织物电子器件的工作与纺织结构的电、力、热特 性密切相关. 织物常包含纤维纱线多层次的结构, 各层 次尺度差异跨越几个数量级. 细观和微观结构与电子 设备结构迥异, 多孔、纤维间移动摩擦、各向异性、 非均质. 此外, 其较易机械变形、有效模量远低于硬质 电子器件及微机电器件、大型变、小应力、热湿膨胀 导致结构不稳定性. 织物电子领域需有针对性地探究 其工作机理并对传统模型进行验证、修改或建立新的 物理模型, 以指导技术研发和产品开发. 此外, 由于其 系统的复杂性和应用环境的多变性等, 目前研究的大 部分产品尚未能同时满足保持电子性能和保留织物优 异的服用性能. 织物电子器件和系统吸引了大量的研 究, 已开始有产业生产, 但其商品化刚刚开始. 


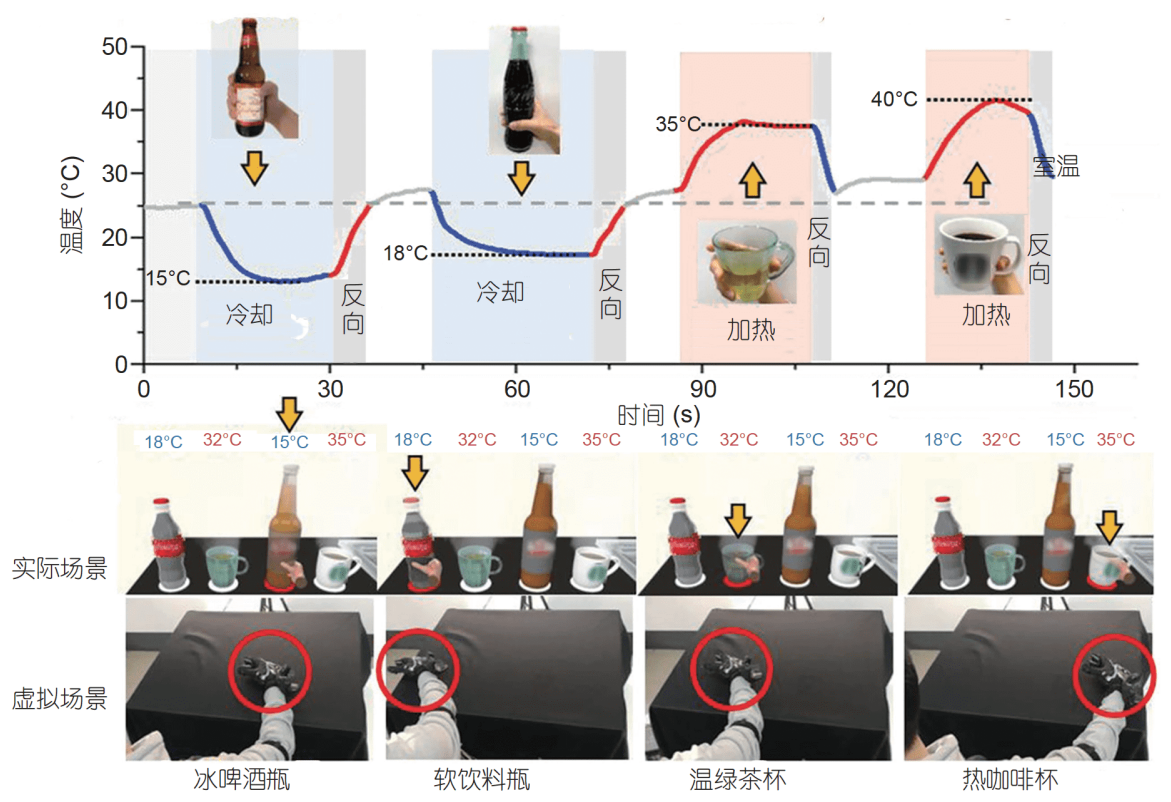

图 5 (网络版彩色)控制微气候的织物及其在虚拟现实中的应用 ${ }^{[118]}$

Figure 5 (Color online) Regenerating of artificial thermal feeling with thermal haptic device when contacting various hot and cold objects in VR space $^{[118]}$

除受本身技术水平限制外，织物电子在应用方面 还有很多挑战. 一个挑战是需要针对特定场景开发系 统. 如可穿戴系统不能影响人体服用舒适度以及纺织 品的其他服用性能. 除此之外, 人工智能算法和专业知 识的结合在应用中有极大潜力. 另一个挑战在于刚柔 并济: 需要将传统的硬质电子器件柔性化、可洗化、 可穿化等. 近年来, 微电子和微机电领域发展迅猛, 设 备集成度越来越高, 体积越来越小. 通过异质集成技术 可将织物电子、集成电路、薄膜电子器件等融合一体, 取长补短, 将是织物电子的一个必然发展方向.

现有织物电子器件性能有待提高, 如传感性能、
可靠性、鲁棒性及寿命一般比刚性微电子器件较差, 需要针对织物的优势研发柔性执行器等新型织物器件; 应促进光电传感器件和系统的微小化集成, 对织物电 子异质混合系统集成的研究需要加强; 增加对设备、 工艺和质检系统的研究，提高织物电子产品生产的一 致性, 逐步建立标准; 应充分重视织物电子系统的应用 场景研究, 包括跨学科的应用场景研究, 如健康医疗、 人工智能、智能防护、运动监测、虚拟现实和增强现 实等, 大力推进织物电子的应用. 能否成功应用决定了 织物电子技术是否能成为主流科技，对该领域的长远 发展极为重要.

\section{参考文献}

1 Li Y, Li Y, Su M, et al. Electronic textile by dyeing method for multiresolution physical kineses monitoring. Adv Electron Mater, 2017, 3: 1700253

2 Wang C, Li X, Gao E, et al. Carbonized silk fabric for ultrastretchable, highly sensitive, and wearable strain sensors. Adv Mater, 2016, 28: 66406648

3 Cai G, Yang M, Xu Z, et al. Flexible and wearable strain sensing fabrics. Chem Eng J, 2017, 325: 396-403

4 RAL Deutsches Institut für Gütesicherung und Kennzeichnung. Medical Compression Hosiery. Berlin: E.V. Beuth Verlag, 2008. RAL-GZ 387/1

5 Wang F, Jiang J, Sun F, et al. Flexible wearable graphene/alginate composite non-woven fabric temperature sensor with high sensitivity and antiinterference. Cellulose, 2019, 27: 2369-2380

6 Trung T Q, Le H S, Dang T M L, et al. Freestanding, fiber-based, wearable temperature sensor with tunable thermal index for healthcare monitoring. Adv Healthc Mater, 2018, 7: e1800074

7 Yun Y J, Hong W G, Kim D Y, et al. E-textile gas sensors composed of molybdenum disulfide and reduced graphene oxide for high response and reliability. Sens Actuat B-Chem, 2017, 248: 829-835 
8 Mirvakili S M, Hunter I W. Artificial muscles: Mechanisms, applications, and challenges. Adv Mater, 2018, 30: 1704407

9 Stoychev G V, Ionov L. Actuating fibers: Design and applications. ACS Appl Mater Interfaces, 2016, 8: 24281-24294

10 Kong L, Chen W. Carbon nanotube and graphene-based bioinspired electrochemical actuators. Adv Mater, 2014, 26: 1025-1043

11 Wehner M, Truby R L, Fitzgerald D J, et al. An integrated design and fabrication strategy for entirely soft, autonomous robots. Nature, 2016, 536: $451-455$

12 Chen D, Pei Q. Electronic muscles and skins: A review of soft sensors and actuators. Chem Rev, 2017, 117: 11239-11268

$13 \mathrm{Lu} \mathrm{C}$, Zhao L, Hu Y, et al. A molecular-regulation strategy towards low-voltage driven, multi degree of freedom IPMC catheters. Chem Commun, 2018, 54: 8733-8736

14 Gong J, Lin H, Dunlop J W C, et al. Hierarchically arranged helical fiber actuators derived from commercial cloth. Adv Mater, 2017, 29: 1605103

15 Cheng H, Hu Y, Zhao F, et al. Moisture-activated torsional graphene-fiber motor. Adv Mater, 2014, 26: 2909-2913

16 Yang X, Wang W, Miao M. Moisture-responsive natural fiber coil-structured artificial muscles. ACS Appl Mater Interfaces, 2018, 10: 3225632264

$17 \mathrm{Hu} \mathrm{Y,} \mathrm{Wu} \mathrm{G,} \mathrm{Lan} \mathrm{T,} \mathrm{et} \mathrm{al.} \mathrm{A} \mathrm{graphene-based} \mathrm{bimorph} \mathrm{structure} \mathrm{for} \mathrm{design} \mathrm{of} \mathrm{high} \mathrm{performance} \mathrm{photoactuators.} \mathrm{Adv} \mathrm{Mater,} \mathrm{2015,} \mathrm{27:} \mathrm{7867-7873}$

$18 \mathrm{Hu} \mathrm{Y,} \mathrm{Chen} \mathrm{W,} \mathrm{Lu} \mathrm{L,} \mathrm{et} \mathrm{al.} \mathrm{Electromechanical} \mathrm{actuation} \mathrm{with} \mathrm{controllable} \mathrm{motion} \mathrm{based} \mathrm{on} \mathrm{a} \mathrm{single-walled} \mathrm{carbon} \mathrm{nanotube} \mathrm{and} \mathrm{natural}$ biopolymer composite. ACS Nano, 2010, 4: 3498-3502

$19 \mathrm{Hu}$ Y, Liu J, Chang L, et al. Electrically and sunlight-driven actuator with versatile biomimetic motions based on rolled carbon nanotube bilayer composite. Adv Funct Mater, 2017, 27: 1704388

20 Copic D, Hart A J. Corrugated paraffin nanocomposite films as large stroke thermal actuators and self-activating thermal interfaces. ACS Appl Mater Interfaces, 2015, 7: 8218-8224

21 Zhang Z, Zhu B, Peng Z, et al. Programmable and thermally hardening composite yarn actuators with a wide range of operating temperature. Adv Mater Technol, 2020, 5: 2000329

22 Arazoe H, Miyajima D, Akaike K, et al. An autonomous actuator driven by fluctuations in ambient humidity. Nat Mater, 2016, 15: 1084-1089

23 Kim S J, Kim O, Park M J. True low-power self-locking soft actuators. Adv Mater, 2018, 30: 1706547

24 Gelebart A H, Jan Mulder D, Varga M, et al. Making waves in a photoactive polymer film. Nature, 2017, 546: 632-636

25 Yu Q, Yang X, Chen Y, et al. Fabrication of light-triggered soft artificial muscles via a mixed-matrix membrane strategy. Angew Chem Int Ed, 2018, 57: 10192-10196

26 Ji M, Jiang N, Chang J, et al. Near-infrared light-driven, highly efficient bilayer actuators based on polydopamine-modified reduced graphene oxide. Adv Funct Mater, 2014, 24: 5412-5419

27 Han B, Zhang Y L, Chen Q D, et al. Carbon-based photothermal actuators. Adv Funct Mater, 2018, 28: 1802235

28 Lan T, Chen W. Hybrid nanoscale organic molecular crystals assembly as a photon-controlled actuator. Angew Chem, 2013, 125: 6624-6628

$29 \mathrm{Wu}$ G, Hu Y, Zhao J, et al. Ordered and active nanochannel electrode design for high-performance electrochemical actuator. Small, 2016, 12: 4986-4992

30 Terasawa N, Asaka K. High-performance PEDOT:PSS/single-walled carbon nanotube/ionic liquid actuators combining electrostatic double-layer and faradaic capacitors. Langmuir, 2016, 32: 7210-7218

$31 \mathrm{Lu} \mathrm{L}$, Liu J, Hu Y, et al. Large volume variation of an anisotropic graphene nanosheet electrochemical-mechanical actuator under low voltage stimulation. Chem Commun, 2012, 48: 3978-3980

32 He S, Chen P, Qiu L, et al. A mechanically actuating carbon-nanotube fiber in response to water and moisture. Angew Chem, 2015, 127: 1509315097

33 Kim S H, Kwon C H, Park K, et al. Bio-inspired, moisture-powered hybrid carbon nanotube yarn muscles. Sci Rep, 2016, 6: 23016

34 Cappello L, Galloway K C, Sanan S, et al. Exploiting textile mechanical anisotropy for fabric-based pneumatic actuators. Soft Robot, 2018, 5: $662-674$

35 Liu Y, An M, Bi Y, et al. Flexible efficient top-emitting organic light-emitting devices on a silk substrate. IEEE Photon J, 2017, 9: 1-6

36 Choi M K, Yang J, Kang K, et al. Wearable red-green-blue quantum dot light-emitting diode array using high-resolution intaglio transfer printing. Nat Commun, 2015, 6: 7149

37 Rein M, Favrod V D, Hou C, et al. Diode fibres for fabric-based optical communications. Nature, 2018, 560: 214-218

38 Ortí E, Bolink H J. Light-emitting fabrics. Nat Photon, 2015, 9: 211-212

39 Choi S, Kwon S, Kim H, et al. Highly flexible and efficient fabric-based organic light-emitting devices for clothing-shaped wearable displays. Sci Rep, 2017, 7: 6424

40 Ok K H, Kim J, Park S R, et al. Ultra-thin and smooth transparent electrode for flexible and leakage-free organic light-emitting diodes. Sci Rep, 2015, 5: 9464

41 Song S, Song B, Cho C H, et al. Textile-fiber-embedded multiluminescent devices: A new approach to soft display systems. Mater Today, 2020, 
32: $46-58$

42 Kim W, Kwon S, Han Y C, et al. Reliable actual fabric-based organic light-emitting diodes: Toward a wearable display. Adv Electron Mater, 2016, 2: 1600220

43 Kwon S, Kim W, Kim H, et al. High luminance fiber-based polymer light-emitting devices by a dip-coating method. Adv Electron Mater, 2015, 1: 1500103

44 Lim Y W, Kwon O E, Kang S M, et al. Built-in haze glass-fabric reinforced siloxane hybrid film for efficient organic light-emitting diodes (OLEDs). Adv Funct Mater, 2018, 28: 1802944

45 Zhang Z, Shi X, Lou H, et al. A stretchable and sensitive light-emitting fabric. J Mater Chem C, 2017, 5: 4139-4144

46 Zhang Z, Zhang Q, Guo K, et al. Flexible electroluminescent fiber fabricated from coaxially wound carbon nanotube sheets. J Mater Chem C, 2015, 3: 5621-5624

47 Lee H E, Lee D, Lee T I, et al. Wireless powered wearable micro light-emitting diodes. Nano Energy, 2019, 55: 454-462

48 Kwon S, Kim H, Choi S, et al. Weavable and highly efficient organic light-emitting fibers for wearable electronics: A scalable, low-temperature process. Nano Lett, 2018, 18: 347-356

49 Zhang Z, Guo K, Li Y, et al. A colour-tunable, weavable fibre-shaped polymer light-emitting electrochemical cell. Nat Photon, 2015, 9: 233-238

50 Zhang Z, Cui L, Shi X, et al. Textile display for electronic and brain-interfaced communications. Adv Mater, 2018, 30: 1800323

51 Chandravanshi A, Rai A, Chaitanya G, et al. Wearable antenna: A critical review. In: 2016 11th International Conference on Industrial and Information Systems. Roorkee: IEEE, 2016. 486-491

52 Yan S, Vandenbosch G A E. Radiation pattern-reconfigurable wearable antenna based on metamaterial structure. Antennas Wirel Propag Lett, 2016, 15: 1715-1718

53 Gong J, Xu B, Tao X. Breath figure micromolding approach for regulating the microstructures of polymeric films for triboelectric nanogenerators. ACS Appl Mater Interfaces, 2017, 9: 4988-4997

$54 \mathrm{Hu}$ Y, Zheng Z. Progress in textile-based triboelectric nanogenerators for smart fabrics. Nano Energy, 2019, 56: 16-24

55 Zhang L, Lin S, Hua T, et al. Fiber-based thermoelectric generators: Materials, device structures, fabrication, characterization, and applications. Adv Energy Mater, 2018, 8: 1700524

56 Zeng W, Tao X M, Lin S, et al. Defect-engineered reduced graphene oxide sheets with high electric conductivity and controlled thermal conductivity for soft and flexible wearable thermoelectric generators. Nano Energy, 2018, 54: 163-174

57 Zhang L S, Yang B, Lin S P, et al. Predicting performance of fiber thermoelectric generator arrays in wearable electronic applications. Nano Energy, 2020, 76: 105117

58 Kim J, Lee J H, Lee J, et al. Research update: Hybrid energy devices combining nanogenerators and energy storage systems for self-charging capability. APL Mater, 2017, 5: 073804

59 Proto A, Penhaker M, Conforto S, et al. Nanogenerators for human body energy harvesting. Trends Biotechnol, 2017, 35: 610-624

$60 \mathrm{Pu} \mathrm{X}, \mathrm{Hu}$ W, Wang Z L. Toward wearable self-charging power systems: The integration of energy-harvesting and storage devices. Small, 2018, 14: 1702817

61 Luo J, Wang Z L. Recent advances in triboelectric nanogenerator based self-charging power systems. Energy Storage Mater, 2019, 23: 617-628

62 Paosangthong W, Torah R, Beeby S. Recent progress on textile-based triboelectric nanogenerators. Nano Energy, 2019, 55: 401-423

63 Dong K, Peng X, Wang Z L. Fiber/fabric-based piezoelectric and triboelectric nanogenerators for flexible/stretchable and wearable electronics and artificial intelligence. Adv Mater, 2020, 32: 1902549

64 Huang L, Lin S, Xu Z, et al. Fiber-based energy conversion devices for human-body energy harvesting. Adv Mater, 2020, 32 : 1902034

65 Shi J, Liu S, Zhang L, et al. Smart textile-integrated microelectronic systems for wearable applications. Adv Mater, 2020, 32: 1901958

66 Tao X. Study of fiber-based wearable energy systems. Acc Chem Res, 2019, 52: 307-315

67 Chen S, Tao X, Zeng W, et al. Quantifying energy harvested from contact-mode hybrid nanogenerators with cascaded piezoelectric and triboelectric units. Adv Energy Mater, 2017, 7: 1601569

68 Chai Z, Zhang N, Sun P, et al. Tailorable and wearable textile devices for solar energy harvesting and simultaneous storage. ACS Nano, 2016, 10: 9201-9207

69 Zeng W, Shu L, Li Q, et al. Fiber-based wearable electronics: A review of materials, fabrication, devices, and applications. Adv Mater, 2014, 26: 5310-5336

$70 \mathrm{Pu}$ X, Song W, Liu M, et al. Wearable power-textiles by integrating fabric triboelectric nanogenerators and fiber-shaped dye-sensitized solar cells. Adv Energy Mater, 2016, 6: 1601048

71 Liu S, Zheng W, Yang B, et al. Triboelectric charge density of porous and deformable fabrics made from polymer fibers. Nano Energy, 2018, 53: 383-390

72 Song J, Yang B, Zeng W, et al. Highly flexible, large-area, and facile textile-based hybrid nanogenerator with cascaded piezoelectric and 
triboelectric units for mechanical energy harvesting. Adv Mater Technol, 2018, 3: 1800016

74 Wang Y, Su S, Cai L, et al. Monolithic integration of all-in-one supercapacitor for 3D electronics. Adv Energy Mater, 2019, 9: 1900037

75 Yang B, Xiong Y, Ma K, et al. Recent advances in wearable textile-based triboelectric generator systems for energy harvesting from human motion. EcoMat, 2020, 2: e12054

76 Liu S, Ma K, Yang B, et al. Textile electronics for VR/AR applications. Adv Funct Mater, 2020, 2007254

77 Falconi C, Martinelli E, Dinatale C, et al. Electronic interfaces. Sens Actuat B-Chem, 2007, 121: 295-329

78 Tao X. Handbook of Smart Textiles. Singapore: Springer, 2016

79 Pulskamp K, Diabaté S, Krug H F. Carbon nanotubes show no sign of acute toxicity but induce intracellular reactive oxygen species in dependence on contaminants. Toxicol Lett, 2007, 168: 58-74

80 Muller J, Huaux F, Fonseca A, et al. Structural defects play a major role in the acute lung toxicity of multiwall carbon nanotubes: Toxicological aspects. Chem Res Toxicol, 2008, 21: 1698-1705

81 Meoli D, May-Plumlee T. Interactive electronic textile development: A review of technologies. J Text Apparel Technol Manage, 2002, 2: 1-12

82 Huo D, Kim M J, Lyu Z, et al. One-dimensional metal nanostructures: From colloidal syntheses to applications. Chem Rev, 2019, 119: 8972-9073

83 Tseghai G B, Mengistie D A, Malengier B, et al. PEDOT:PSS-based conductive textiles and their applications. Sensors, 2020, 20: 1881

$84 \mathrm{Yu} \mathrm{C}$, An J, Zhou R, et al. Microstructure ercapacitors. Microstructure design of carbonaceous fibers: A promising strategy toward highperformance weaveable/wearable supercapacitors. Small, 2020, 16: 2000653

85 Wang F, Liu S, Shu L, et al. Low-dimensional carbon based sensors and sensing network for wearable health and environmental monitoring. Carbon, 2017, 121: 353-367

86 Ke S H, Xue Q W, Pang C Y, et al. Printing the ultra-long Ag nanowires inks onto the flexible textile substrate for stretchable electronics. Nanomaterials, 2019, 9: 686

87 Mao Y, Zhu M, Wang W, et al. Well-defined silver conductive pattern fabricated on polyester fabric by screen printing a dopamine surface modifier followed by electroless plating. Soft Matter, 2018, 14: 1260-1269

88 Karim N, Afroj S, Malandraki A, et al. All inkjet-printed graphene-based conductive patterns for wearable e-textile applications. J Mater Chem C, 2017, 5: 11640-11648

89 Yli-Pentti A. Electroplating and electroless plating. In: Hashmi S, Batalha G F, Van Tyne C J, et al., eds. Comprehensive Materials Processing. Amsterdam: Elsevier, 2014. 277-306

90 Park S J, Ko T J, Yoon J, et al. Copper circuit patterning on polymer using selective surface modification and electroless plating. Appl Surf Sci, 2017, 396: 1678-1684

91 Sha W, Wu X, Keong K G. Electroless Copper and Nickel-Phosphorus Plating: Processing, Characterisation and Modelling. Amsterdam: Elsevier, 2011

92 Allison L, Hoxie S, Andrew T L. Towards seamlessly-integrated textile electronics: Methods to coat fabrics and fibers with conducting polymers for electronic applications. Chem Commun, 2017, 53: 7182-7193

93 Zhang M, Wang C, Wang H, et al. Carbonized cotton fabric for high-performance wearable strain sensors. Adv Funct Mater, 2017, 27: 1604795

94 Frank E, Hermanutz F, Buchmeiser M R. Carbon fibers: Precursors, manufacturing, and properties. Macromol Mater Eng, 2012, 297: 493-501

95 Yan W, Page A, Nguyen-Dang T, et al. Advanced multimaterial electronic and optoelectronic fibers and textiles. Adv Mater, 2019, 31: 1802348

96 Liu M, Pu X, Jiang C, et al. Large-area all-textile pressure sensors for monitoring human motion and physiological signals. Adv Mater, 2017, 29: 1703700

97 Afroj S, Karim N, Wang Z, et al. Engineering graphene flakes for wearable textile sensors via highly scalable and ultrafast yarn dyeing technique. ACS Nano, 2019, 13: 3847-3857

98 Kim S J, We J H, Cho B J. A wearable thermoelectric generator fabricated on a glass fabric. Energy Environ Sci, 2014, 7: 1959-1965

99 Li Q, Chen H, Ran Z Y, et al. Full fabric sensing network with large deformation for continuous detection of skin temperature. Smart Mater Struct, 2018, 27: 105017

100 Li Q, Tao X M. Three-dimensionally deformable, highly stretchable, permeable, durable and washable fabric circuit boards. Proc R Soc A: Math Phys Eng Sci, 2014, 470: 20140472

101 Ismar E, Tao X, Rault F, et al. Towards embroidered circuit board from conductive yarns for e-textiles. IEEE Access, 2020, 8: 155329

102 Molla M T I, Goodman S, Schleif N, et al. Surface-mount manufacturing for e-textile circuits. In: Proceedings of the 2017 ACM International Symposium on Wearable Computers. New York: Association for Computing Machinery, 2017

103 Hughes-Riley T, Dias T. Developing an acoustic sensing yarn for health surveillance in a military setting. Sensors, 2018, 18: 1590

104 Trindade I G, Martins F, Miguel R, et al. Design and integration of wearable devices in textiles. Sensors Transd, 2014, 183: 42-47

105 Leśnikowski J. Research on poppers used as electrical connectors in high speed textile transmission lines. Autex Res J, 2016, 16: 228-235 
106 Linz T, Vieroth R, Dils C, et al. Embroidered interconnections and encapsulation for electronics in textiles for wearable electronics applications. Adv Sci Technol, 2008, 60: 85-94

107 Cui H, Li D, Fan Q. Reliability of flexible electrically conductive adhesives. Polym Adv Technol, 2013, 24: 114-117

108 Islam S. Textile CAD analysis for warp and weft patterning. J Text Sci Eng, 2018, 8: 1000332

109 Rathnayake A S. Development of the core technology for the creation of electronically-active, smart yarn. Doctor Dissertation. Nottingham: Nottingham Trent University, 2015

110 Zhang Y Q, Luo S L. Safety testing requirements for smart wearable textiles (in Chinese). Prog Texile Sci Technol, 2018, (5): 17-19 [张宇群, 罗 胜利. 智能可穿戴纺织品的安全性测试要求. 纺织科技进展, 2018, (5): 17-19]

111 Min S D, Yun Y, Shin H. Simplified structural textile respiration sensor based on capacitive pressure sensing method. IEEE Sens J, 2014, 14: 3245-3251

112 Samy L, Huang M C, Liu J J, et al. Unobtrusive sleep stage identification using a pressure-sensitive bed sheet. IEEE Sens J, 2014, 14: 2092-2101

113 Wang X, Tao X, So R C H, et al. Monitoring elbow isometric contraction by novel wearable fabric sensing device. Smart Mater Struct, 2016, 25: 125022

114 Blecha T, Soukup R, Kaspar P, et al. Smart firefighter protective suit-functional blocks and technologies. In: Proceedings of the 2018 IEEE International Conference on Semiconductor Electronics (ICSE). Kuala Lumpur: IEEE, 2018

115 Reiffenrath M, Hoerr M, Gries T, et al. Smart protective clothing for law enforcement personnel. Mater Sci Text Cloth Technol, 2014, 9: 64-68

116 Shu L. Wearable body area sensor networks for continuous dynamic health monitoring in daily activities: Case study of intelligent footwear system. Doctor Dissertation. Hong Kong: The Hong Kong Polytechnic University, 2012

117 Choi S, Yoon K, Lee S, et al. Conductive hierarchical hairy fibers for highly sensitive, stretchable, and water-resistant multimodal gesturedistinguishable sensor, VR applications. Adv Funct Mater, 2019, 29: 1905808

118 Lee J, Sul H, Lee W, et al. Stretchable skin-like cooling/heating device for reconstruction of artificial thermal sensation in virtual reality. Adv Funct Mater, 2020, 30: 1909171

119 Vortmann L M, Kroll F, Putze F. EEG-based classification of internally- and externally-directed attention in an augmented reality paradigm. Front Hum Neurosci, 2019, 13: 348 


\title{
Recent advances, scientific issues, key technologies and perspective of textile electronics
}

\author{
Xiaoming Tao ${ }^{1,2^{*}}$, Su Liu ${ }^{1,2}$, Bao Yang ${ }^{1,2}$, Lisha Zhang ${ }^{1,2}$, Kitming $\mathrm{Ma}^{1,2}$, Lin Shu ${ }^{3} \&$ Fei Wang ${ }^{1,2}$ \\ ${ }^{1}$ Research Center for Smart Wearable Technology, Hong Kong Polytechnic University, Hong Kong, China; \\ ${ }^{2}$ Institute of Textiles and Clothing, Hong Kong Polytechnic University, Hong Kong, China; \\ ${ }^{3}$ School of Electronic and Information Engineering, South China University of Technology, Guangzhou 510640, China \\ * Corresponding author, E-mail: xiao-ming.tao@polyu.edu.hk
}

Textile electronics refer to the fibers or fiber assemblies (yarn, fabric, or clothing) possessing electronic functions, including generation, transmittance, evaluation, and modulation of electrons. It includes devices and systems. Since textile electronics are light, flexible, breathable, and functional, it exhibits great potentials in a wide range of applications. However, the noticeable progress of textile electronics has been focused on large-area, flexible devices with several functions, such as sensation, actuation, display, antenna, and energy harvesting and storage. The current textile electronic system is achieved by hybrids of microelectronic chips and textile electronic devices. They corporate together to provide complicated functions of sensation, actuation, communication, analysis, control, learning, memory, and adaption. The textile electronic system is an important branch of wearable electronics. This article provides a comprehensive review and analysis of textile electronic devices, electronic system integration, and applications.

Firstly, it focuses on the development and technology of a number of textile electronic devices, which include sensors, actuators, displays, antennas, energy storage, and generators. This section introduces the functions, classification, performance, and limitations of textile electronic devices. In the textile electronic system, the signal module, microprocessor, data storage, and communication module now cannot be realized on textile due to the technology limitation. Therefore, it is necessary to incorporate the relevant microelectronic chips and micro-electro-mechanic devices into the textile to achieve system-on-fabric.

The next section of this review describes and analyzes the research gaps of textile electronics in wearable applications. The key requirement is that textile electronics should deliver advanced electronic functions without deteriorating the textile properties. In addition, textile electronics also need to face strict requirements on biocompatibility, laundering, and comfortability. We then elaborate on the major scientific issues in several dimensions, covering design, synthesis, characterization of flexible electronic materials, multi-physics and multi-scale modeling of textile electronics devices and systems, test standardization of textile electronic devices and systems, and safety issues of textile electronics.

Then, we discuss the present core technologies of textile electronics in various aspects. The electronic materials include intrinsic conductive materials that include metal in different dimensions, conductive and semiconducting polymer, and carbon derivatives. The additive fabrication technology covers printing techniques, coating, physical vapor deposition, chemical vapor deposition, in situ polymerization, carbonization, and others. At the device level, the fabrication technology can be separated into single multicomponent fiber electronics, or fiber assemblies, or heterogeneous assembly of microelectronic chips in the textile structure, elaborated with examples. In addition, we discuss different connection integration methods, such as embroidery, weaving, and knitting. We also discuss the software development for textile electronics, optical fiber system requirements, and test standards for textile electronics.

The last section elaborates on potential applications of textile electronics. In online healthcare and individualized medical treatments, textile electronics provide real-time monitoring and wireless feedback of human physiological and physical signals, such as temperature, electrocardiogram (ECG), electromyography (EMG), respiration, gait, posture, etc. or provide intervention and treatments. It breaks down the geographic limitation and increases the effectiveness of treatment. In sports, based on the obtained real-time data of individuals and AI models of a large population, wearable textile electronic systems may offer advice on training so that efficiency can be enhanced, and injury is avoided. The textile electronics can detect potential environmental hazards, such as toxic substances and impact by falling objects or vehicles. In addition, the intimate contact characteristics of textile electronics render more immersion in virtual and augmented reality experiments to users by real-time feedback on physiological parameters, and by additional stimulation, such as haptic, olfactory, and microclimate. It broadens the scope of applications of augmented reality and virtual reality in online education, online design, online gaming.

textile electronics, textile electronic systems, wearable textile, functional materials, scientific issues, key technologies

doi: 10.1360/TB-2020-1402 